\title{
SISTEMA INTELIGENTE PARA LA GESTIÓN DE UN CENTRO DE DISTRIBUCIÓN BASADO EN LA IDENTIFICACIÓN DE PRODUCTOS
}

\author{
Intelligent system for the management of an \\ distribution center based on the product identification
}

\section{EPISTEMUS}

ISSN: 2007-8196 (electrónico)

ISSN: 2007-4530 (impresa)

\section{Julio César Encinas Alvarado ${ }^{1}$}

Juan Pablo Soto Barrera ${ }^{2}$

Roberto Núñez González²

Andrés García Higuera ${ }^{3}$

Recibido: 27 de mayo de 2017,

Aceptado: 22 de septiembre de 2017

Autor de Correspondencia:

Dr. Julio César Soto Barrera

Correo: jencinas@uabc.edu.mx

\section{Resumen}

La globalización, aunada a otros factores, ha originado un conjunto de transformaciones en las decisiones sobre la producción, dando paso a la reestructuración de los mercados y de la organización de la distribución. Esto ha originado una demanda en el desarrollo de sistemas que permitan un mejor control, manipulación, seguimiento y distribución de productos en entornos distribuidos. Dichos entornos favorecen la subdivisión del sistema total en subsistemas más pequeños y abarcables, con la capacidad de interactuar unos con otros, con la intención de alcanzar un objetivo colectivo.

En este trabajo se propone un sistema automático-inteligente basado en control distribuido que permite la identificación y gestión de pedidos mediante el uso de agentes inteligentes y la Identificación por Radiofrecuencia (RFID, por sus siglas en inglés: Radio Frequency Identification) para la gestión de un centro de distribución.

\section{Abstract}

Globalization, together with other factors, has led to a series of transformations in production decisions, giving way to the restructuring of markets and the organization of distribution. This has led to a demand in the development of systems that allow better control, manipulation, monitoring and distribution of products in distributed environments. These environments favor the subdivision of the total system into smaller and more encompassing subsystems, with the ability to interact with each other, with the intention of achieving a collective objective.

This paper proposes an automatic-intelligent system based on distributed control that allows the identification and management of orders through the use of intelligent agents and Radio Frequency Identification (RFID, for its acronym in English: Radio Frequency Identification) for the management of a distribution center.

1 Universidad Autónoma de Baja California / Facultad de Ingeniería - Mexicali / Correo:jencinas@uabc.edu.mx

2 Universidad de Sonora / Departamento de Matemáticas - Hermosillo / Correo: jpsoto@gauss.mat.uson.mx

3 Universidad de Castilla-La Mancha / E.T.S. de Ingenieros Industriales - Ciudad Real (España) /Correo: andres.garcia@uclm.es 


\section{INTRODUCCIÓN}

Dadas las nuevas exigencias en los mercados globales, el sector de producción industrial ha tenido que evolucionar para enfrentar esta situación de tal manera que hoy se habla de las nuevas industrias y estilos de gestión logística empresarial. Debido a este entorno de alta competitividad, un conjunto de transformaciones han dado paso a la reestructuración de los mercados y de la organización de la distribución. Obligando a las compañías a mejorar día a día, en términos de calidad, respuesta, agilidad y flexibilidad, siendo éstas, cualidades decisivas para permanecer en un negocio [1]. Esta situación conlleva múltiples consecuencias para la industria, por ejemplo, la reducción del ciclo de vida de los productos, el aumento de la diversidad, la rápida satisfacción de la demanda, la reducción de costos excesivos, entre otros [2]. Es por esto, que en modelos de negocios tan complejos y cambiantes se requiere que la información del entorno sea lo más exacta y fiable posible, para que el sistema de gestión pueda tomar decisiones adecuadas y precisas [3].

Actualmente la mayoría de las empresas utilizan el código de barras para la trazabilidad y seguimiento de sus productos. Sin embargo, uno de los principales problemas de este mecanismo radica en su lectura, ya que, ocasionalmente, las etiquetas están dañadas, mal colocadas, o parcialmente despegadas. En estos casos, los lectores no pueden realizar correctamente su función, por lo que afecta a la hora de gestionar la información en tiempo real de acuerdo con la precisión del sistema. Debido a esto, consideramos que la Identificación por Radiofrecuencia, está llamada a hacerse con el lugar que ha sido ocupado por el código de barras durante más de 30 años. Si además, a la Identificación por Radiofrecuencia se dota de un sistema de información es posible identificar la información relativa a un producto. Por otra parte, si el sistema de información es gestionado por agentes inteligentes que faciliten el procesamiento y manejo de la información de manera distribuida, se garantizaría el desarrollo de sistemas más capaces en cuanto al manejo de la información en tiempo real. Teniendo esto en cuenta, en este trabajo diseñamos un sistema automáticointeligente de control distribuido basado en la integración de la tecnología RFID y el uso de agentes inteligentes (Intelligent Management Systems, RFID-IMS). Para dar más claridad, la metodología propuesta se describe mediante su aplicación a un caso concreto basado en un centro de distribución real representado en una plataforma experimental. Una descripción detallada de la plataforma antes mencionada puede encontrarse en [5].En la sección 2 se describe la metodología de gestión propuesta (RFIDIMS). Posteriormente, en la sección 3, se describe la implementación del sistema de control en una plataforma experimental. En la sección 4 se presenta la arquitectura utilizada, así como la integración de la tecnología RFID con los agentes inteligentes. Finalmente, las conclusiones y trabajos futuros son presentadas en la sección 5 .

\section{METODOLOGÍA RFID-IMS}

El sistema de gestión RFID-IMS consta de una importante parte de hardware basada en la identificación por radiofrecuencia. Durante el proceso de producción que se lleva acabo en el centro de distribución, los productos son identificados por antenas ubicadas en puntos estratégicos distribuidos por la planta, además del área de entradas y salidas del centro de distribución. La gestión de dicha información se lleva acabo a un bajo nivel por la red de PLCs (por sus siglas en inglés: Programmable Logic Controller) que controla el área de elaboración o producción de órdenes y a un nivel alto por el ERP (por sus siglas en inglés: Enterprise Resource Planning).

Según [5], las capacidades funcionales de los sistemas ERP están basadas en el sistema proveedor de información y la arquitectura utilizada en la implementación del sistema ERP. Es decir, que el éxito de la solución generada por el ERP depende, en gran parte, de la veracidad de la información guardada en sus bases de datos. Por lo tanto, para poder tener un proceso de toma de decisiones correcto, es necesario una tecnología generadora de información confiable que mantenga las bases de datos con información actualizada.

La arquitectura propuesta está basada en un Sistemas Multi-agente (SMA). Este tipo de arquitectura le proporciona al sistema ERP la capacidad de enviar y recibir la información generada por el sistema RFID-IS y la red de PLCs. De esta forma, el ERP a través del sistema RFIDIS puede visualizar la situación de la planta y el estado de avance de las órdenes de producción. Esto conlleva tres aportaciones importantes:

- Visibilidad del centro de distribución.

- Ajuste dinámico de los niveles de prioridad.

- Libramiento del canal de comunicación entre la red de PLCs y el ERP.

El sistema ERP está obligado a mantener una constante comunicación con los distintos elementos que componen los módulos; en especial con el módulo de cadena de suministro. Por tanto, el envío y recepción de información entre el ERP y el elemento controlador de la planta, que en este caso es la red de PLCs, es constante. Básicamente las comunicaciones consisten en el envío de las órdenes de trabajo, niveles de prioridad, cambios de nivel de prioridad, finalización de elaboración de la orden, etc.

Sin embargo, hay situaciones en las que el cliente presiona al centro de distribución por su pedido, estos casos conllevan un crecimiento (hostigamiento) en la comunicación del ERP hacia la red de PLCs que muchas veces termina saturando los canales de comunicación. 
Además, algunas veces este hostigamiento por parte del ERP hacia el controlador del centro de distribución es innecesario debido a que el palé o pedido se encuentra en una zona o situación, como puede ser un cuello de botella, donde los PLCs no pueden realizar ninguna acción para agilizar su envío.

Para hacer frente a este problema, en la propuesta se ha dotado al sistema ERP de una visibilidad directa del centro de distribución, por medio de la tecnología RFID. En otras palabras, el ERP tiene acceso a la información generada por los lectores RFID instalados en distintos puntos estratégicos a lo largo del centro (Figura1). De esta manera, el ERP sabe cuándo es útil hacer un cambio de prioridad urgente o en qué área del centro de distribución debe de presionar.

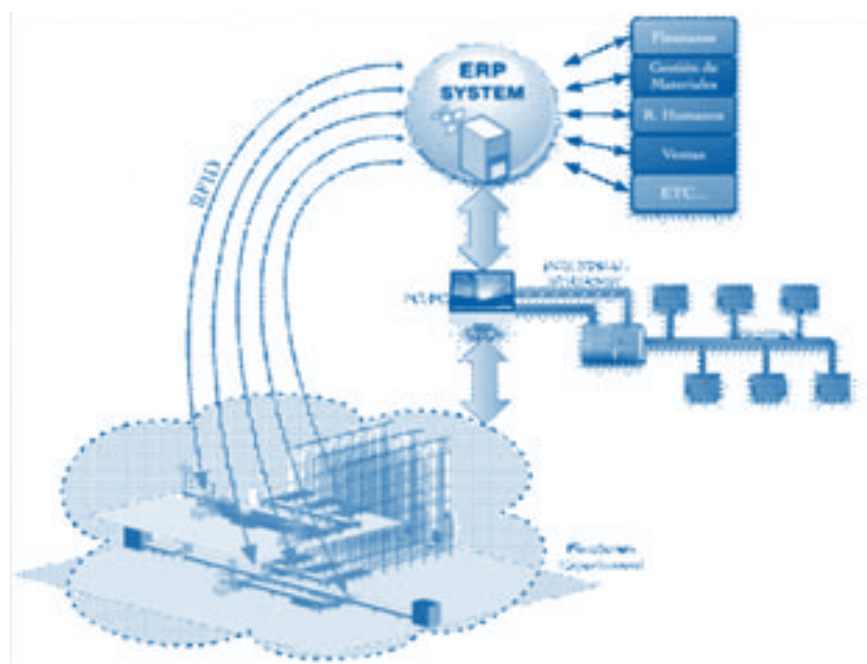

Figura 1. Esquema general de comunicaciones en el centro de distribución

Es muy importante que los agentes encargados de la gestión del centro de distribución, tengan la capacidad de hacer ajustes en los niveles de prioridad en función de las demandas de los distribuidores, clientes y otros factores que se detallarán más adelante. De esta forma, pueden organizar la elaboración de las órdenes de producción mediante el movimiento dinámico de los niveles de prioridad de cada orden, inclusive de cada palé, además, de proveer al sistema con una gran flexibilidad.

Por ejemplo, supongamos que se tiene en proceso de elaboración un pedido de nivel medio y a mitad del pedido entra una orden de un cliente de mayor importancia, evidentemente con una prioridad más alta. Entonces al encontrarse los palés de distintas órdenes en los cruces de las cintas trasportadoras, se realizará una comparativa del nivel de prioridad, dando paso al de nivel más alto. Esto nos produce ciertas ventajas como el aumento de flexibilidad del centro, disminución tiempo de respuesta y de elaboración de pedidos.
Sin embargo, el movimiento de prioridades debe de ser dinámico, es decir, que vaya cambiando respecto al progreso en la elaboración del pedido. Para esto, se propone un modelo matemático donde la prioridad va cambiando en función de distintos factores y de una forma dinámica. De tal modo que conforme la orden de trabajo es cumplimentada el nivel de prioridad para los últimos palés va ascendiendo y así evitando atascos y retrasos.

\section{SISTEMA DE CONTROL E IMPLEMENTACIÓN}

La implementación del sistema de control para el centro de distribución ha sido realizada en varias partes. Si bien, el desarrollo de cada una de las partes que integran el sistema, siempre se ha realizado de forma coordinada con el resto. Teniendo en cuenta que la mayor parte de la metodología RFID-IMS está implementada en la red de PLCs, el sistema ERP y la parte hardware de RFID, la migración de esta tecnología a cualquier empresa no supone un gran costo [6].

Se trata de un sistema automático de control distribuido basado en agentes en el que cada una de las partes que lo integran es independiente de las demás. La implementación de este tipo de técnicas proporciona una gran optimización en los sistemas, ya que son un conjunto de pequeños esfuerzos, que sumados provocan que el conjunto funcione de manera sólida y eficiente. Además, en caso de fallo o caída de alguna de las partes que forman el sistema, únicamente dejaría de funcionar la parte afectada, mientras que el resto del sistema seguirá funcionando con normalidad, evitando así, un fenómeno de cascada del fallo.

\section{CADENA DE DATOS}

Como se ha comentado anteriormente, el centro de distribución está controlado por una red de PLCs. Entre el sistema de gestión y el de control, existen una serie de comunicaciones, en las que se envían y reciben las cadenas de información que contienen las órdenes. En dichas órdenes se incorpora únicamente la información necesaria para el correcto funcionamiento del centro de distribución.

Las citadas órdenes presentan una determinada estructura (Figura 2), en la que aparecen codificados, de forma eficiente, los datos necesarios. Se trata de una cadena binaria de 4 Bytes que incorpora: identificación del palé dentro del sistema de control, origen y destino del mismo, un conjunto de flags, habilitados para la escritura de posibles errores producidos durante el cumplimiento de la orden, y un espacio reservado para definir la prioridad de cada orden. Esto servirá al sistema de control implementado en los PLC para llevar a cabo correctamente su tarea, y será determinante a la hora de actuar en ciertas situaciones críticas, que se producen en la actividad cotidiana del centro de distribución. 


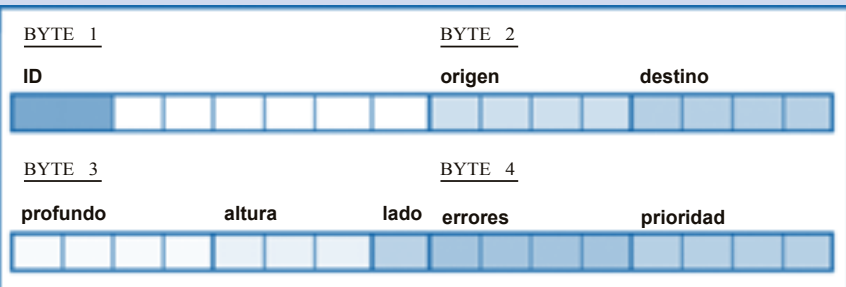

Figura 2. Formato de la cadena de datos (Fuente: [7])

\section{RED DE PLCS}

Para la implementación del sistema de control del centro de distribución, se van a emplear una serie de PLCS SIMATIC S7 de Siemens conectados mediante una red PROFIBUSDP. Se tienen entonces 7PLCs; un maestro y 6 esclavos. El maestro es un Siemens SIMATIC S7 300 CPU 313C-2DP, el cual va acoplado con un módulo de red Siemens modelo CP343-1, el cual le permite interactuar vía Internet con otros entes. Los esclavos son 6 Siemens SIMATIC S7 200 CPU 224, conectados a través de módulos EM-227 de PROFIBUS-DP.

El elemento más importante de la red es el PLC Master, ya que permite la comunicación de la red con el exterior, y por tanto con el resto de los controladores, a través de una red LAN (Figura 3).

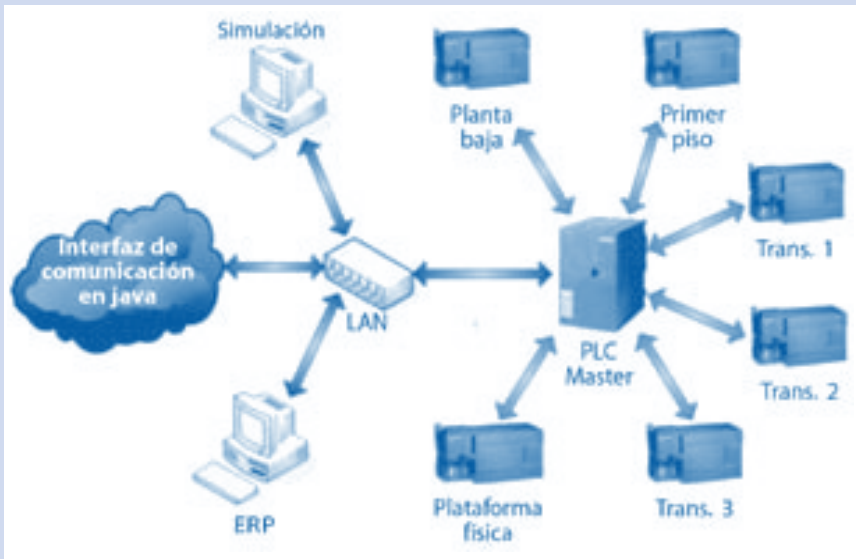

Figura 3. Esquema general del funcionamiento del sistema (Fuente: [7])

La principal función de este PLC es la de establecer comunicación con cada uno de los PLC esclavos y con el interfaz de comunicación. Es decir, gestionar la información y las comunicaciones entre cada uno de los PLCs que forman la red y el exterior de la misma, y por tanto con el resto del sistema de gestión y control del centro de distribución.

EI PLC Master es el encargado de recoger las cadenas de datos (órdenes) que llegan desde el sistema de gestión, almacenándolas en el Bloque de Datos (DB) de recepción. Después de analizar la orden, la envía al PLC esclavo que corresponda. Previo a la llegada de la orden al PLC esclavo correspondiente, la orden es transferida a otro DB, que hace como bandeja de entrada de cada esclavo. Una vez que la orden es procesada por el PLC esclavo, la envía a su bandeja de salida (su DB correspondiente). En ese momento, el Master se encarga de recoger la orden que es devuelta por el PLC esclavo, y de redireccionar a otro PLC o a la interfaz de comunicación, para informar al sistema de gestión sobre la ejecución de la orden, según el caso.

\section{INTERFAZ CON LA SIMULACIÓN}

Dado que el sistema de control se encuentra implementado en una red de PLCs y el centro de distribución es simulado en su mayor parte, resulta imprescindible la creación de un interfaz de comunicación para conectar ambas partes y poder controlar la planta. Se ha desarrollado un interfaz en Java, utilizando Eclipse, mediante el uso de sockets que comunican las señales de los PLCs (entradas y salidas), con el software de simulación Grasp 10 (Figura 4). El interfaz se conecta con ambas partes utilizando una red LAN, lo que aporta una gran flexibilidad al sistema, ya que las partes pueden encontrarse en distintas ubicaciones físicas. De este modo, dicha interfaz se conecta por un lado con el módulo LAN de la red de PLCs, y por otro lado con la computadora en la que se ejecuta la simulación.

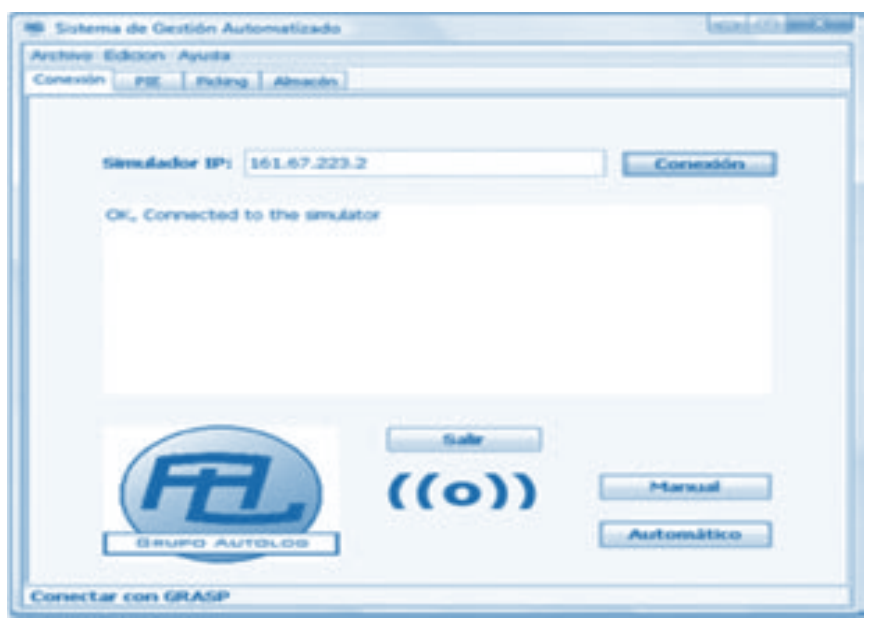

Figura 4. Ventana de conexión del interfaz

Esta interfaz consiste en la interpretación y correspondencia del conjunto de entradasy salidas de la red de PLCs, con una serie de mensajes de texto interpretables y generados por el software de simulación. Para ello, este interfaz se basa en el uso de bases de datos MySQL, en las que se almacenan las relaciones entre las direcciones de E/S de los PLCs y los eventos de la simulación, ya sean instrucciones de comando hacia la simulación o los mensajes generados por la misma.

\section{ARQUITECTURA MULTI-AGENTE}

Uno de los objetivos de los SMA es que los agentes tengan la capacidad de negociar entre ellos para enfrentar situaciones de conflicto [8]. Basados en los conceptos 
definidos en [9], se ha diseñado una arquitectura multiagente para su aplicación en el centro de distribución. El sistema está estructurado en tres niveles reactivo, deliberativo e interfase (Figura 5).

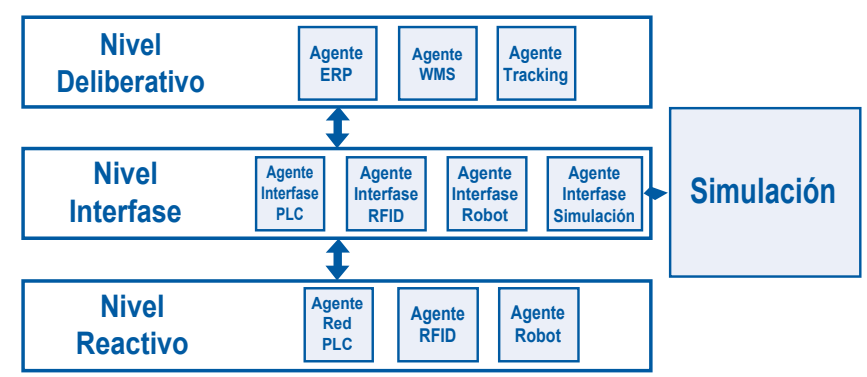

Figura 5. Arquitectura basada en Agentes(Fuente: [4])

\section{NIVEL REACTIVO}

En este nivel los PLCs controlan el modelo físico y la simulación. Los agentes que componen este nivel tienen la habilidad de percibir cambios en el ambiente y reaccionar automáticamente a ellos sin necesidad de ser analizadas.

Agente Red PLC (ARP): Este agente es el encargado de recibir un paquete de información del nivel deliberativo que contiene entre otras cosas, la identificación del palé, origen, destino, nivel de prioridad y los almacena en DBs.

La comparación del nivel de prioridad de cada palé en el puntos de cruces, trazabilidad de palés y movimiento de cargas son tareas llevadas a cabo por los sub-agentes (PLCs) que componen la ARP. Los componentes de este módulo son:

- Master PLC: se encarga de procesar la información procedente del sistema de gestión y repartirla a cada uno de los PLC esclavos, según corresponda.

- PLC 1: es el encargado de realizar el control de la planta baja del centro de distribución.

- PLC 2: éste se encarga del control de la segunda planta del centro de distribución.

- PLC 3, 4 y 5: cada uno de ellos se encarga del control respectivo de los tres transelevadores de los que dispone el almacén.

- PLC 6: se encarga del control de los muelles de carga y descarga del centro de distribución en coordinación con la simulación.

Agente RFID: este agente asigna una identificación única a cada uno de los palés que entran en la planta y envía esa identificación al nivel deliberativo.

Agente Robot: este agente es responsable del movimiento de los palés miniatura desde la cinta trasportadora hasta la estantería y viceversa.

\section{NIVEL INTERFASE}

Este nivel es muy importante ya que la efectividad del sistema de gestión depende, en gran parte de la exactitud y rapidez de la información procesada en este nivel. La función principal es transferir información del nivel reactivo al nivel deliberativo y viceversa. Es decir, funciona como un puente de comunicaciones entre ambos niveles. En este nivel, los siguientes agentes se encuentran conectados a través de Ethernet:

Agente interfase PLC: este agente maneja toda la información y las comunicaciones entre los agentes que componen la red de PLCs y los otros módulos.

Agente interfase RFID: estos lectores incorporan módulos internos de red desarrollados en el grupo autolog, para ser capaces de comunicarse vía Ethernet.

Agente interfase Robot: el robot cuenta con una tarjeta de red industrial y es controlado a través de un software hecho en Java basado en interfaces de programación de aplicaciones (API, por sus siglas en inglés: ApplicationProgramming Interface) de Motoman.

Agente interfase Simulación:a través de sockets, un software desarrollado en Java coordina la comunicación entre el parte de control y la simulación.

\section{NIVEL DELIBERATIVO}

En este nivel, los agentes tienen la habilidad de recibir y procesar información sobre su entorno y según sus creencias y deseos, decidir cuál es el mejor plan de acción para alcanzar los objetivos especificados. En este nivel podemos encontrar:

Agente ERP: Este agente se encarga del procesamiento de las acciones que se aplicarán para alcanzar objetivos específicos. Recibe órdenes de los clientes, genera la planificación en coordinación con el Agente WMS y calcula el nivel de prioridad de las órdenes. Este agente ha sido implementado en el ERP "Hermes" creado sobre la plataforma Velneo 6.4.1.

Agente WMS: Este agente gestiona el almacén automático simulado. Se encarga de asignar la locación final del palé en el almacén automático, de acuerdo con diferentes factores.

Agente Tracking: Este agente está encargado de dar un seguimiento a las órdenes. De esta manera, si hay algún retraso en alguna orden de producción, debe modificar el nivel de prioridad de cada palé para poder cumplimentar a tiempo la orden.

\section{CÁLCULO DE PRIORIDADES}

La decisión de mandar un palé a cierta locación o asignarle una prioridad específica se realiza en una coordinación entre los agentes ARP y ERP. Por ejemplo, si un palé $k_{j i}$ de una orden $\mathrm{K}_{\mathrm{j}} \mathrm{y}$ un palé $\mathrm{k}_{\mathrm{rl}}$ de la orden de producción $\mathrm{K}_{\mathrm{r}}$ llegan al mismo tiempo a un cruce de cintas trasportadoras. El ARP debe ser capaz de seleccionar el palé con mayor prioridad.

RFID-IMS a través de su implementación en el ERP, asigna un nivel de prioridad dependiendo de la orden de producción a la que pertenecen. El nivel de prioridad es determinado por la ecuación (1). Esta ecuación está inspirada en un modelo matemático, utilizado en el cálculo del valor de reputación/confianza de un agente 


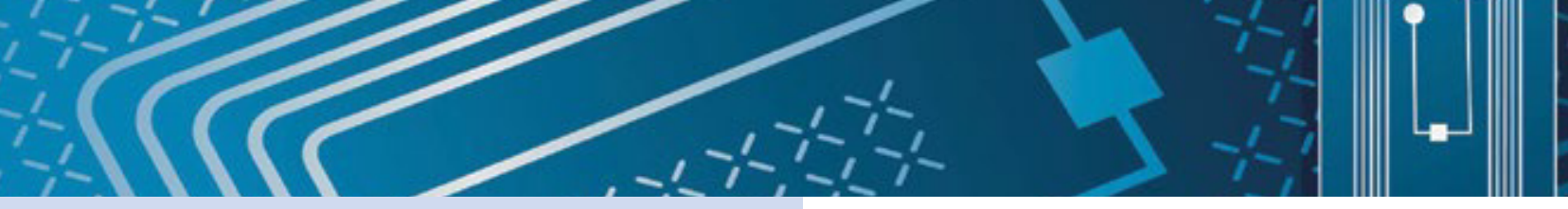

dentro de un SMA [10], [11]. En este trabajo, este modelo ha sido adaptado para el cálculo del nivel de prioridad de un palé dentro de una comunidad de palés (órdenes de producción).

$$
P_{k_{j i}}=\mathrm{w}_{c} C_{K_{j}}+w_{d} D_{K_{j}}+\mathrm{w}_{\mathrm{t}} \mathrm{\top}_{K_{j}}+\frac{\sum_{i=1}^{n} S_{k_{j i}}}{n}
$$

Donde $\mathrm{P}_{\mathrm{k}_{\mathrm{ji}}}$ denota la prioridad. $\mathrm{C}_{\mathrm{k}_{\mathrm{j}}}$ es el grado de importancia de un cliente. La compañía, en este caso centro de distribución, debe proveer este valor; el cual debe estar comprendido: $0 \leq C_{\mathrm{K}_{j}} \leq 1$. $D_{\mathrm{K} j}$ es el valor asignado a la fecha de entrega, al igual que el valor anterior, es definido por la compañía y su valor comprende entre $0 \leq D_{K_{r}} \leq 1$. $T_{K}$ denota el valor asignado a la fecha de recibo o entrada y su valor es de $0 \leq T_{K_{j}} \leq 1$. El último término de la ecuación nos indica el nivel de progreso de la orden. Es decir, hasta qué punto de la orden se ha elaborado. Este valor es el resultado de la sumatoria de los factores $S_{\mathrm{kji}}$ todo eso dividido entre el número total de palés de la orden correspondiente. $S_{k_{j i}}$ es una variable binaria que es 0 cuando el palé $k_{j i}$ no ha sido embarcado y 1 en el caso contrario.

Finalmente, $w_{c}, w_{d}$ y $w_{t}$ son pesos asignados respectivamente a las variables $C_{k_{j}}, D_{k_{j}} y T_{k_{j}}$. Con esto, el valor de prioridad puede ser ajustada a la necesidad de la organización. Los pesos deberán tener un valor de 0 a 1 dependiendo de la política del centro de distribución.

El nivel de prioridad está ubicado en las cadenas de datos adjuntas a cada palé y puede ser actualizado a través de Ethernet, siguiendo las instrucciones del nivel deliberativo.

\section{CONCLUSIONES}

En el mercado actual, las industrias están obligadas a definir nuevas estrategias que les permitan competir en el mercado, acompañadas de herramientas de gestión adecuadas que garanticen su materialización; sin ellas, los esfuerzos serán inútiles. En consecuencia, las tecnologías de control deben adaptarse a estos nuevos entornos de producción y tener acceso automático a la información. La facilidad en el acceso a la información, así como la capacidad de recolección de datos de tiempo real, se convierten en requisitos esenciales para un manejo eficaz de la cadena de suministro.

En este artículo se describe la implementación de la metodología RFID-IMS en un centro de distribución. La metodología propuesta dota al sistema con la capacidad de reaccionar de una manera flexible ante cambios inesperados. Esto se logra mediante la aplicación de un sistema capaz de proveer datos precisos utilizando la tecnología RFID, junto a un control negociador basado en agentes. Por otro lado, la metodología RFID-IMS responde efectivamente a requerimientos dinámicos, mostrando beneficios inmediatos en distintos procesos de distribución. Primero el sistema es capaz de gestionar distintos lotes al mismo tiempo; incrementando el número de órdenes terminadas sobre las que son elaboradas siguiendo mecanismos de planeación tradicionales. Otro beneficio importante es la preparación de órdenes dependiendo de su nivel de prioridad, misma que ha sido el talón de Aquiles de muchos centros de distribución. Este proceso de preparación de órdenes se mejora debido al correcto uso de los recursos en el centro de distribución.

El desempeño del área de embarque también mejora por la reducción de los errores en los procesos de carga y descarga. Estos errores (Ej. la devolución de productos) son muy importantes, debido al alto costo que generan a la compañía además de la afectación negativa de la imagen.

\section{BIBLIOGRÁFIA}

1) Leitão, P., (2009) Agent-based distributed manufacturing control: A state-of-the-art survey, Engineering Applications of Artificial Intelligence, 22, 979-991.

2) McFarlane, D., Marík, V., Valckenaers, P. (2005).Intelligent Control in the Manufacturing Supply Chain.IEEE IntelligentSystems, 20(1), 24-27.

3) García, A. \&Cenjor, A., (2007). Sistema Heterárquico de Control Basado en Agentes para Sistemas de Fabricación, Revista iberoamericana de automática e informática industrial (RIAI), 4,83 .

4) Encinas J.C., García A., de las Morenas J. (2010). Improvements in operations management applied to a 3D simulation connected to a physical platform. Journal of Intelligent Manufacturing, Springer, Netherlands. DOI: 10.1007/s10845010-0441-0.

5) Chan, S. (1999). Architecture Choices for ERP Systems, Proceedings 5th Americas Conference on Information Sys- tems, Milwaukee, pp. 210-212.

6) Encinas J.C., García F.P., de las Morenas J., García A. (2009). "Operations Management based on RFID-IMSII". Proc. of the 23rd European Conference on Modelling and Simulation (ECMS'09). Madrid, Spain. June 9-12.

7) Blanco, Jesus; García, Andrés; De Las Morenas, Javier; García, Pablo; García-Escribano, Javier. (2010).Application of Distributed Control Theories and RFID in a Miniature Distribution Center.The Third International EURASIP Workshop on RFID Technology.6-7 septiembre. La Manga del Mar Menor (Cartagena). ISBN: 97884-96997-47-9.

8) Wooldridge M., G. Weiss, y P. Ciancarini. (2002). Agent-Oriented Software Engineering II.LNCS vol. 2222. Springer-Verlag: Berlin, Germany.

9) Bonasso, R.P., Firby, J., Gat, E., Kortenkamp, D., Millar, D., Snack, M. (1997). A Proven Three-tiered Architecture for Programming Autonomous Robots.Journal of Experimental and Theoretical Artificial Intelligence, vol. 9, no. 2

10) Soto, J. P., Vizcaíno, A., Portillo-Rodríguez, J., Piattini, M. (2008). Recommending Trustworthy Knowledge in KMS by Using Agents. Software and Data Technologies, Springer-Verlag Berlin Heidelberg, 22, 297-309,

11) Soto, J.P., Vizcaíno, A., Portillo-Rodriguez, J., Piattini, M., Kusche, O. (2009). A Two-Layer Multi-agent Architecture to Facilitate Knowledge Sharing within Communities of Practice.Inteligencia Artificial (IA), 13(42), 46-54. 